\title{
ELECTRONIC WORD-OF-MOUTH E A SUA INFLUÊNCIA NA INTENÇÃO DE COMPRA DOS UTILIZADORES DO FACEBOOK
}

\section{ELECTRONIC WORD-OF-MOUTH AND ITS INFLUENCE ON FACEBOOK USERS 'PURCHASE INTENTION}

\author{
Miguel Teixeira ${ }^{1}$ \\ António Andrade ${ }^{1}$ \\ Carla Martins $^{1}$
}

\begin{abstract}
Resumo: Os websites de social media são principalmente impulsionados por conteúdos gerados pelos indivíduos e a sua popularidade exponenciou a capacidade de influência das comunicações interpessoais sobre produtos ou serviços no comportamento dos consumidores entre redes sociais online. Este estudo examinou os determinantes do Electronic Word-of-Mouth (eWOM) transmitido pela lista de contactos no Facebook que influenciam a intenção de compra dos consumidores. $O$ modelo testado é adaptado do modelo Information Acceptance (IACM) de Erkan e Evans (2016). Os objetivos de pesquisa consistem na investigação de características da informação (qualidade $e$ credibilidade de informação), do recetor (atitude em relação à informação e necessidades de informação) e do emissor (credibilidade da fonte) que exercem maior impacto na influência do eWOM no comportamento do consumidor. A utilidade da informação foi considerada como antecedente chave das respostas de comportamento (adoção da informação e intenção de compra). Dados recolhidos através de um questionário online a 294 utilizadores do Facebook residentes em Portugal foram analisados através da técnica de modelação de equações estruturais baseada no método de mínimos quadrados parciais (PLS-SEM). As características do recetor demonstraram ter o maior nível de influência na intenção de compra. A qualidade da informação não demonstrou efeito sobre a intenção de compra. Adicionalmente, a credibilidade da informação apresentou um efeito mediador total sobre a qualidade da informação e a credibilidade da fonte na perceção de utilidade de informação.
\end{abstract}

Palavras-chave: eWOM; intenção de compra; Facebook; modelo de equações estruturais (SEM); mínimos quadrados parciais (PLS)

${ }^{1}$ Católica Porto Business School: aandrade@ porto.ucp.pt 


\begin{abstract}
Social media websites are driven mainly by usergenerated content, and their popularity augmented the interpersonal communications' ability to influence consumer behavior regarding products or services between online social networks. This study examined the determinants of Electronic Word-of-Mouth (eWOM) transmitted by Facebook friends which influence consumers' purchasing intention. The model used is adapted from Information Acceptance Model (IAM) by Erkan and Evans (2016). Research objectives consist on the investigation of characteristics of the information (information quality and credibility), the receiver (attitude towards information and needs of information) and the sender (source credibility), which have the greatest impact on eWOM's influence on consumer behavior. Information usefulness was considered a key antecedent of the consumer's responses (information adoption and purchase intention). Data collected through an online questionnaire on 294 Facebook users residing in Portugal were analyzed using the partial least squares structural equation modeling (PLS-SEM). Receiver's characteristics show the highest impact on purchase intention. Information quality doesn't present any effect on purchase intention. Additionally, information credibility appears to have a total mediating effect over information quality and source credibility on information usefulness.
\end{abstract}

Keywords: eWOM; purchase intention; Facebook; structural equation modeling (SEM); partial least squares (PLS).

\title{
INTRODUÇÃO
}

Uma peça de informação tem "valor informacional" quando é capaz de influenciar a tomada de decisão (Brown, Broderick, \& Lee, 2007). A comunicação Word-of-Mouth (WOM) é um meio de transmissão deste tipo de informação, sendo reconhecido na literatura a sua importante influência sobre os consumidores. Ao processo de difusão de WOM através da internet dá-se o nome de electronic Word-of-Mouth (eWOM), que pode ser definido como: "qualquer grau ou combinação de comentários, recomendações, ou declarações positivas, negativas ou neutras, sobre empresas, marcas, produtos ou serviços, discutidos ou partilhados entre consumidores em formatos digitais ou eletrónicos" (Rodgers \& Wang, 2011).

É relevante o estudo de como informações sobre consumo, transmitidas entre indivíduos desinteressados, afetam a intenção de compra, sobretudo com o advento da internet (Goldsmith, 2006; HennigThurau et al., 2010) que possibilitou uma difusão de informação maior em volume, alcance e dispersão, e mais persistente no que se refere ao 
seu formato, disponibilidade e clareza, para referir apenas alguns fatores (King, Racherla, \& Bush, 2014).

O eWOM pode ocorrer através de uma grande variedade de meios, como, por exemplo, email, mensagens instantâneas, blogs, fóruns, salas de chat, websites de ecommerce, websites de reviews e websites de social media (Cheung \& Thadani, 2012; Goldsmith, 2006). Existe um grande número de estudos em diferentes contextos que sugerem que estas informações expostas online constituem uma importante fonte facilitadora e influenciadora na tomada de decisão dos consumidores (e.g., Chang \& Wu, 2014; Cheung, Lee, \& Rabjohn, 2008; Cheung, Luo, Sia, \& Chen, 2009; Lee \& Shin, 2014; Wang, Yu, \& Wei, 2012; Zhang; \& Watts, 2008; Zhang, Zhao, Cheung, \& Lee, 2014), contudo a literatura encontrase ainda relativamente limitada quanto à influência do eWOM nos websites de social media (Balaji, Wei, \& Chong, 2016; Cheung \& Thadani, 2012).

Os websites de social media - onde os indivíduos trocam informação revelando as suas identidades reais - têm ganho cada vez mais importância e possuem características interessantes para a geração de eWOM, ao fomentarem a partilha e disseminação de informação (Erkan \& Evans, 2016; Kim \& Johnson, 2016; Yan et al., 2016). Um estudo efetuado pela PricewaterhouseCoopers (2017) indica que, progressivamente, a população global dá menos importância à publicidade tradicional, preferindo obter informação autêntica provinda de amigos e outros utilizadores. Destes websites, de momento, o Facebook é o mais popular e dos mais utilizados como fonte de informação de apoio para a tomada de decisão de compra (PricewaterhouseCoopers, 2017), contando com um total de 2,07 biliões de utilizadores ativos mensalmente (Facebook, 2017).

Assim, para colmatar a aparente insuficiência de cobertura dos efeitos do eWOM na tomada de decisão dos consumidores, particularmente sentida no contexto do Facebook, este estudo tem como objetivo identificar os determinantes do eWOM transmitido pela lista de contactos no Facebook que influenciam a intenção de compra dos consumidores. Para esse efeito é usado o modelo Information Acceptance Model (IACM) como referência, recentemente desenvolvido por Erkan e Evans (2016) especificamente para a examinação do eWOM em websites de social media. Este modelo aborda a influência das características do recetor e da informação eWOM (da mensagem) na intenção de compra dos consumidores. No presente estudo foi ainda acrescentado ao IACM um fator de modo a ter em conta as características da fonte, permitindo 
uma análise mais completa, englobando as três principais dimensões da influência da comunicação sobre os indivíduos de acordo com o modelo Yale: a mensagem, a fonte, e o recetor (Chang \& Wu, 2014; Cheung et al., 2009).

\section{REVISÃO DE LITERATURA}

\section{1 eWOM em websites de social media}

Nos últimos anos, os websites de social media têm apresentado um grande crescimento, passando a ter uma força considerável como meios de comunicação. Estes atraem milhões de utilizadores, sendo que atualmente os consumidores despendem uma porção significativa do seu tempo nestas plataformas (Alhidari \& Paswan, 2015).

Estes websites são impulsionados por conteúdos gerados pelos utilizadores (Obar \& Wildman, 2015), disponibilizados para serem visualizados, copiados, modificados e disseminados para além do controlo de quem os gera (Macdonald, 2012). Os utilizadores interagem assim entre si de forma direta, instantânea e expandida (Balaji et al., 2016) para a partilha e consumo de informação e ideias (Alhidari \& Paswan, 2015; Obar \& Wildman, 2015).

Devido às características colaborativas e sociais destas plataformas, elas são consideradas um potencial veículo fortemente apropriado para o eWOM (Chen, Tang, Wu, \& Jheng, 2014; Chu \& Kim, 2011; Erkan \& Evans, 2016) e de facto verifica-se um aumento na proeminência do eWOM com a abundância de informação gerada e difundida nestas plataformas por uma base crescente de utilizadores (Alhidari \& Paswan, 2015). É sugerido que este aumento terá potencialmente alterado a abordagem dos consumidores quanto à procura de informação e tomada de decisões de compra (Chu \& Kim, 2011).

Antes do advento dos websites de social media, uma das principais diferenças consideradas entre o WOM tradicional e o eWOM era o anonimato implícito (Erkan \& Evans, 2016; King et al., 2014; Kozinets, de Valck, Wojnicki, \& Wilner, 2010), podendo este ser uma grande vantagem ao conceder a capacidade de comunicação sobre um produto ou serviço de forma genuína, assim como o maior inconveniente devido à possibilidade do fornecimento de informações falsas sem repercussões (Abbasi \& Liu, 2013; King et al., 2014). Em contraste, nos websites de social media os indivíduos criam perfis pessoais que fornecem informação aos outros utilizadores relativamente aos donos dos perfis (Erkan \& Evans, 2016). Comparativamente a informações de fontes 
anónimas que ocorrem noutras plataformas, os contactos de social media são membros das redes pessoais dos consumidores, podendo estes ser percebidos como mais credíveis e fidedignos, tendo assim o eWOM o potencial para ser mais influente (Chu \& Choi, 2011; Chu \& Kim, 2011).

Apesar de tanto o eWOM como os websites de social media terem recebido uma atenção considerável nos últimos anos por parte de investigadores, a literatura quanto à influência do eWOM nestas plataformas na tomada de decisão dos indivíduos encontra-se ainda relativamente limitada (Balaji et al., 2016; Cheung \& Thadani, 2012).

No Quadro 1, é apresentada uma síntese dos estudos realizados na última década que examinam o impacto do eWOM na tomada de decisão dos indivíduos.

\section{Quadro 1}

Estudos de eWOM na tomada de decisão dos indivíduos

\begin{tabular}{|c|c|c|c|c|}
\hline & Estudo & Impacto estudado & Plataforma & $\begin{array}{c}\text { Método de } \\
\text { investigação }\end{array}$ \\
\hline 2007 & $\begin{array}{l}\text { (Park, Lee, \& Han, } \\
\text { 2007) } \\
\text { (Riegner, 2007) }\end{array}$ & $\begin{array}{l}\text { Intenção de } \\
\text { compra } \\
\text { Decisão de } \\
\text { compra }\end{array}$ & $\begin{array}{l}\text { Website de } \\
\text { ecommerce } \\
*\end{array}$ & $\begin{array}{l}\text { Experimental } \\
\text { Inquérito }\end{array}$ \\
\hline 2008 & $\begin{array}{l}\text { (Awad \& } \\
\text { Ragowsky, 2008) }\end{array}$ & $\begin{array}{l}\text { Intenção de } \\
\text { compra online }\end{array}$ & $\begin{array}{l}\text { Websites de } \\
\text { ecommerce }\end{array}$ & Inquérito \\
\hline - & $\begin{array}{l}\text { (Cheung et al., } \\
\text { 2008) }\end{array}$ & $\begin{array}{l}\text { Adoção da } \\
\text { informação } \\
\text { eWOM }\end{array}$ & $\begin{array}{l}\text { Website de } \\
\text { review } \\
\text { (Openrice) }\end{array}$ & Inquérito \\
\hline - & (Park \& Kim, 2008) & $\begin{array}{l}\text { Intenção de } \\
\text { compra }\end{array}$ & $*$ & Experimental \\
\hline - & $\begin{array}{l}\text { (Zhang \& Watts, } \\
\text { 2008) }\end{array}$ & $\begin{array}{l}\text { Adoção da } \\
\text { informação } \\
\text { eWOM }\end{array}$ & Fóruns & Inquérito \\
\hline 2009 & $\begin{array}{l}\text { (Cheung et al., } \\
\text { 2009) }\end{array}$ & $\begin{array}{l}\text { Adoção da } \\
\text { informação } \\
\text { eWOM }\end{array}$ & $\begin{array}{l}\text { Fórum } \\
\text { (Myetone) }\end{array}$ & Inquérito \\
\hline - & $\begin{array}{l}\text { (Doh \& Hwang, } \\
\text { 2009) }\end{array}$ & $\begin{array}{l}\text { Intenção de } \\
\text { compra }\end{array}$ & $*$ & Experimental \\
\hline 2010 & $\begin{array}{l}\text { (Liu \& Zhang, } \\
\text { 2010) }\end{array}$ & $\begin{array}{l}\text { Adoção da } \\
\text { informação } \\
\text { eWOM }\end{array}$ & $\begin{array}{l}\text { Website de } \\
\text { ecommerce }\end{array}$ & $\begin{array}{l}\text { Inquérito } \\
\text { presencial }\end{array}$ \\
\hline - & $\begin{array}{l}\text { (Prendergast, Ko, \& } \\
\text { Yuen, 2010) }\end{array}$ & $\begin{array}{l}\text { Intenção de } \\
\text { compra }\end{array}$ & Fóruns & Inquérito \\
\hline 2012 & (Fan \& Miao, 2012) & $\begin{array}{l}\text { Intenção de } \\
\text { compra }\end{array}$ & 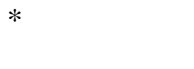 & Inquérito \\
\hline - & (Wang et al., 2012) & $\begin{array}{l}\text { Intenção de } \\
\text { compra }\end{array}$ & $\begin{array}{l}\text { Websites de } \\
\text { social media }\end{array}$ & Inquérito \\
\hline
\end{tabular}




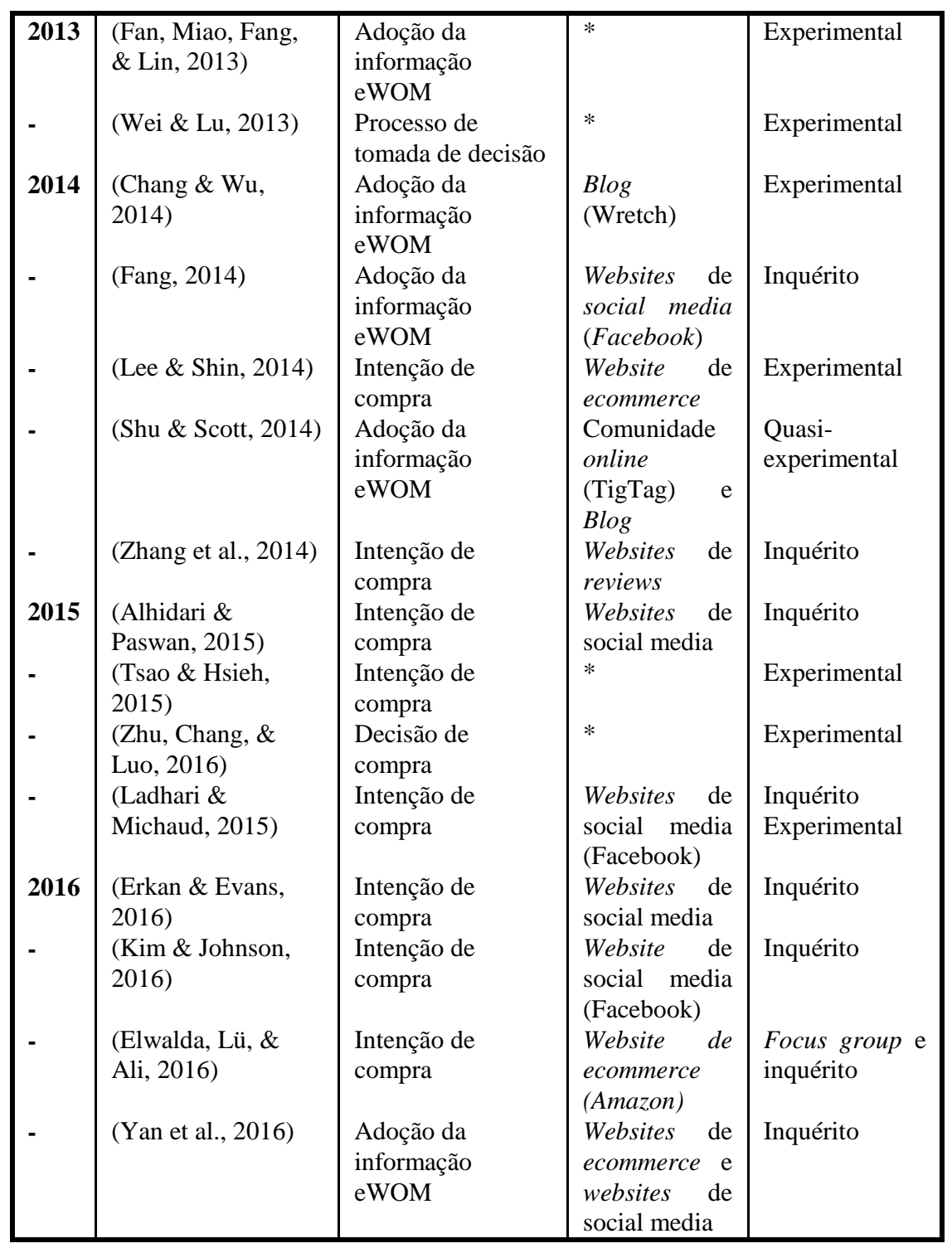

* websites em geral

\section{2. eWOM no Facebook}

No caso do Facebook, os utilizadores são informados, através da newsfeed, de posts feitos por indivíduos da sua lista de contactos e de muitas outras atividades, sendo que estes podem interagir e responder a todo esse tipo de conteúdo através de comentários, reações (gosto, etc...) 
ou partilhas (Caers et al., 2013). Isto resulta na possibilidade de uma grande difusão do eWOM a partir do círculo social dos indivíduos (Chu \& Kim, 2011). Com a grande popularização desta plataforma, esta tornou-se uma importante fonte adicional de informação, com os consumidores a fazerem cada vez mais uso de conteúdos aí postados para avaliar produtos e serviços (PricewaterhouseCoopers, 2017; Yan et al., 2016).

O newsfeed ${ }^{2}$ visa mostrar aos utilizadores os conteúdos que estes consideram mais relevantes (Facebook, 2016a), sendo uma ferramenta em constante evolução. Em 2016, foi atualizada para dar prioridade aos posts efetuados pelos "amigos" mais próximos em detrimento de conteúdo postado pelas fan pages ${ }^{3}$ (Facebook, 2016b), sendo assim o eWOM efetuado pela lista de contactos mais importante para os utilizadores desta plataforma e, consequentemente, para as organizações. De acordo com a própria organização, "quando as pessoas vêm conteúdo em que estão interessadas, estas são mais propensas a gastar tempo no newsfeed" (Facebook, 2016a), tornando-se também maior o volume de conteúdo lido, sugerindo maior probabilidade de observação de eWOM.

A literatura referente ao eWOM no contexto do Facebook é ainda escassa, principalmente quanto ao impacto na tomada de decisão dos consumidores. Dos estudos encontrados, Ladhari e Michaud (2015) foram os primeiros a examinar a influência do eWOM realizado pela lista de contactos do Facebook no processo de tomada de decisão, contudo estes não distinguem os determinantes do eWOM que influenciam a resposta de comportamento do consumidor. Kim e Johnson (2016) debruçaram-se sobre as influências do eWOM partilhado em fan pages no Facebook, revelando que a qualidade da informação influencia positivamente a intenção de compra dos recetores, embora não considerando outras características da informação nem do comportamento dos consumidores em relação à informação, entendidas por alguns autores como importantes para a compreensão do fenómeno do eWOM no contexto deste tipo de plataformas (e.g.,, Erkan \& Evans, 2016; Knoll, 2016; Wolny \& Mueller, 2013).

\subsection{Modelo IACM e modelos relacionados}

A Theory of Reasoned Action (TRA) (Fig. 1) é um modelo desenvolvido por Fishbein e Ajzen (1975) que procura explicar as

\footnotetext{
${ }^{2}$ https://www.facebook.com/help/327131014036297/

${ }^{3}$ https://www.facebook.com/help/282489752085908/
} 
relações entre atitudes e comportamentos, postulando que intenções de comportamento são decididas por normas subjetivas e de atitude (extraído de (Erkan \& Evans, 2016)). É utilizado para prever as intenções e o comportamento dos indivíduos, tendo em conta possíveis mudanças de intenção e critérios de comportamento e atitudes pré-existentes (Sheppard, Hartwick, \& Warshaw, 1988).

\section{Figura 1}

Modelo Theory of Reasoned Action (TRA) (Davis, Bagozzi, \& Warshaw, 1989).

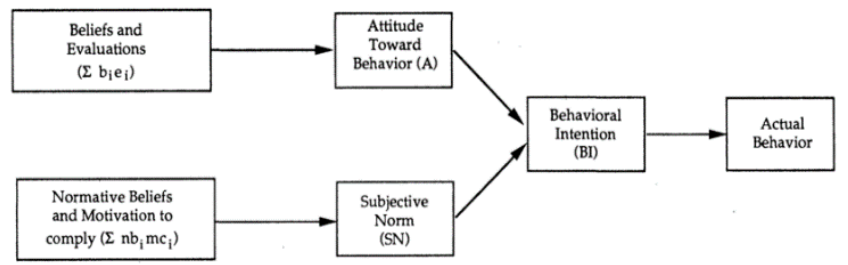

O Technology Acceptance Model (TAM) (Fig. 2) é uma extensão do TRA aplicada aos sistemas de informação, tendo sido desenvolvido para explicar como utilizadores aceitam e utilizam, ou seja, adotam, uma nova tecnologia, considerando que a utilidade e facilidade de utilização percebida afetam os comportamentos dos mesmos (Davis, 1989; Erkan \& Evans, 2016).

\section{Figura 2}

Modelo Technology Acceptance Model (TAM) (Davis, Bagozzi, \& Warshaw, 1989).

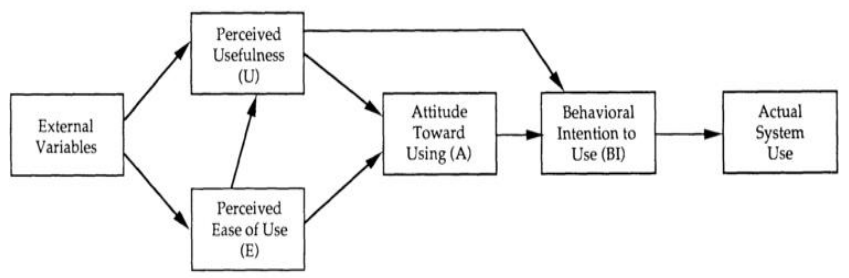

No contexto do eWOM, o TRA é usado para explicar as relações entre a atitude do consumidor, a intenção de compra e a compra (Cheung $\&$ Thadani, 2012), tendo o TAM também já sido aplicado para explicar a adoção da informação (Erkan \& Evans, 2016). No entanto, o TAM é criticado pelo seu poder explanatório limitado (Elwalda et al., 2016; 
Erkan \& Evans, 2016). Enquanto este modelo é um primeiro passo útil para compreender como as intenções em relação a uma mensagem são formadas, este não foi desenvolvido para responder a questões sobre o processo da influência em si (Sussman \& Siegal, 2003). Mais especificamente, este foca na tecnologia (utilização do computador) e descarta processos sociais essenciais relativos à elaboração da informação (Erkan \& Evans, 2016).

Nesse sentido, Sussman e Siegal (2003) desenvolvem o Information Adoption Model (IAM) (Fig. 3), que é uma integração do TAM com o Elaboration Likelihood Model (ELM). O ELM, elaborado por Petty e Cacioppo (1986), postula que uma mensagem pode influenciar as atitudes e comportamentos das pessoas em duas formas: central (racional) refere-se à natureza dos argumentos na mensagem - e periférica (emocional) - refere-se a questões que não estão diretamente relacionadas com o assunto da mensagem (Cheung et al., 2008). Assim, a qualidade da informação (central) e a credibilidade da fonte (periférica) são considerados determinantes chave para os consumidores decidirem se a informação é útil, sendo postulado que a utilidade da informação desempenha um papel importante na adoção da informação (Sussman \& Siegal, 2003). Visto que a influência da informação pode variar de pessoa para pessoa (Cheung et al., 2008; Sussman \& Siegal, 2003), o modelo desenvolvido por Sussman eSiegal (IAM) visa explicar como os indivíduos são afetados pela informação, de modo a adotá-la, adaptado para o contexto de plataformas de comunicação mediadas pelo computador, sendo assim um modelo adequado para estudos de eWOM (Cheung et al., 2008; Zhang; \& Watts, 2008; Zhu et al., 2016).

\section{Figura 3}

Modelo Information Adoption Model (IAM) (Sussman \& Siegal, 2003)

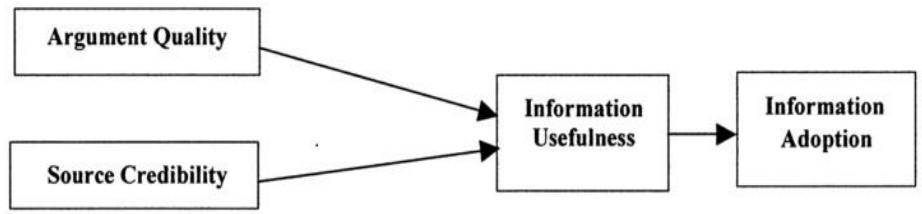

Erkan e Evans (2016) criticam o IAM por ser limitado às características da informação, argumentando que a influência do eWOM não só depende destas características, como também do comportamento 
dos consumidores em relação à informação. Posto isto, estes autores propõem o Information Acceptance Model (IACM) (Fig. 4), uma tentativa de desenvolvimento de um modelo mais robusto, integrando o IAM - que explica a influência das características da informação (qualidade, credibilidade e utilidade da informação) na adoção da mesma - com componentes relacionados do TRA (atitude em relação à informação) - que representa as características do recetor, mais especificamente, expressando o comportamento do consumidor em relação à informação e a relação deste com a intenção de compra. Adicionalmente, este modelo inclui as necessidades de informação também como uma característica do recetor em relação à informação, sendo considerado um determinante importante da perceção da utilidade da informação e, consequentemente, da adoção da informação e intenção de compra. Assim, este modelo baseia-se em seis variáveis: qualidade da informação, credibilidade da informação, necessidades de informação, atitudes em relação à informação, utilidade da informação e adoção da informação.

\section{Figura 4}

Modelo Information Acceptance Model (IACM) (Erkan \& Evans, 2016)

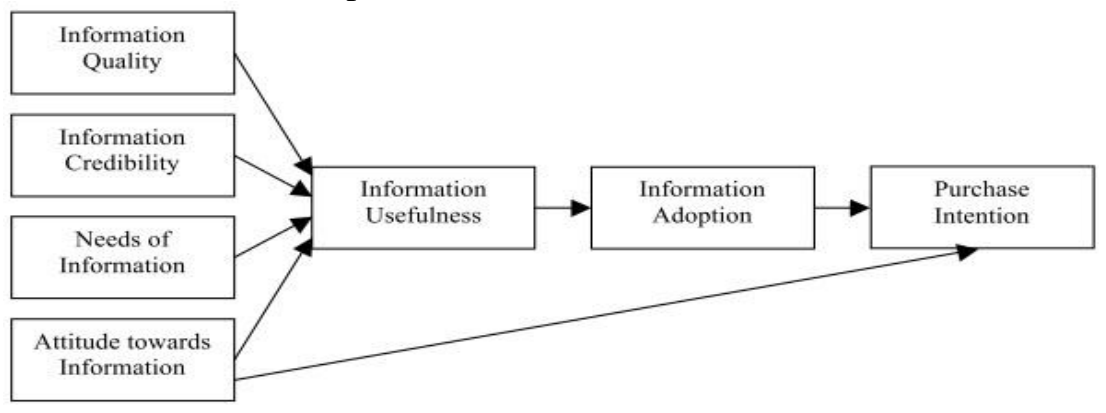

\section{Modelo Conceptual e Hipóteses}

O modelo Yale de 1953, um dos principais modelos de comunicação social, postula que a influência ocorre através de três componentes principais: a mensagem, a fonte e o recetor (Chang \& Wu, 2014; Cheung et al., 2009). O eWOM representa uma forma de comunicação social de conteúdo (estímulo ou informação), envolvendo indivíduos que recebem a informação (recetores) e indivíduos que a partilham (emissores ou fonte) (Cheung \& Thadani, 2012). Atendendo a esta perspetiva, é possível classificar os determinantes do eWOM a serem explorados no 
modelo conceptual utilizado no presente estudo como características da informação, da fonte ou do recetor, como exposto no Quadro 2.

O modelo IACM de Erkan e Evans (2016), de modo a examinar a influência do eWOM na intenção de compra dos consumidores, é desenvolvido através da extensão do modelo IAM (Sussman \& Siegal, 2003) com a integração do modelo TRA de Fishbein e Ajzen (1975). O modelo considera tanto as características da informação (qualidade e credibilidade da informação), como as características do recetor (necessidades de informação e atitudes em relação à informação), denominadas por estes autores de comportamento dos consumidores em relação à informação, desconsiderando portanto as características da fonte - credibilidade da fonte no modelo IAM (Sussman \& Siegal, 2003).

No estudo realizado por Chang e Wu (2014), é sugerido que a credibilidade da fonte exerce influência na adoção da informação apenas de forma indireta, através da credibilidade da informação. No entanto, Chang e Wu (2014) não consideram a utilidade da informação no modelo, quando também esta abordagem é contestada por Sussman e Siegal (2003), que argumentam que a perceção da utilidade da informação serve de mediador no processo de influência entre a adoção da informação e as variáveis da qualidade da informação e credibilidade da fonte.

Deste modo, no presente estudo, é utilizado o modelo IACM (Erkan \& Evans, 2016), sendo acrescentada uma variável considerada no modelo IAM (Sussman \& Siegal, 2003) - credibilidade da fonte - de modo a verificar se esta apresenta uma relação direta com a utilidade da informação, e/ou indireta através da credibilidade da informação. Tendo em conta a perspetiva do modelo Yale, o acréscimo desta variável contribui para uma análise mais completa, considerando as três principais dimensões da influência da comunicação. O Quadro 2 mostra a classificação das variáveis do modelo conceptual usado neste estudo.

Assim, no presente estudo, o modelo conceptual (Figura 5) contempla as sete hipóteses do modelo IACM original (Erkan \& Evans, 2016) e tem em conta três hipóteses adicionais de investigação, nomeadamente H5, H9a e H9b. 


\section{Quadro 2}

Classificação das variáveis do modelo conceptual utilizado

\begin{tabular}{ll}
\hline Classificação & Variáveis \\
\hline Características da Informação & Qualidade da Informação \\
& Credibilidade da Informação \\
\hline Características da Fonte & Credibilidade da Fonte \\
\hline Características do Recetor & Necessidades de Informação \\
& Atitude em Relação à Informação \\
\hline Variáveis Dependentes & Utilidade da Informação \\
& Adoção da Informação \\
& Intenção de Compra \\
\hline
\end{tabular}

\section{A. Adoção da Informação}

A adoção da informação refere-se à medida em que os indivíduos aceitam a informação eWOM que recebem, como significativa (Zhang \& Watts, 2008), e que a utilizarão, como ajuda, na tomada de decisão de compra (Fan et al., 2013). De acordo com Erkan e Evans (2016), a adoção da informação eWOM que é recebida por parte dos consumidores leva a uma maior intenção de compra dos mesmos. Deste modo, segundo o modelo IACM:

H1: Adoção da informação eWOM influencia positivamente a intenção de compra dos consumidores.

\section{B. Utilidade da Informação}

Utilidade da informação refere-se à perceção do indivíduo do quão útil poderá ser uma nova informação para ajudá-lo numa melhor tomada de decisão (Cheung et al., 2008; Erkan \& Evans, 2016). Deste modo, de acordo com Sussman e Siegal (2003), a utilidade da informação é um determinante chave da adoção da informação eWOM, pois se um indivíduo pensar que a informação é útil, terá maior intenção de adotá-la (Cheung et al., 2008). Assim, de acordo com Erkan e Evans (2016):

H2: Utilidade da informação eWOM influencia positivamente a adoção da informação eWOM.

\section{Qualidade da Informação}

De acordo com Sussman e Siegal (2003), a qualidade da informação recebida pode ser um importante determinante do nível da influência dessa informação. Portanto, em concordância com o modelo IACM:

H3: Qualidade da informação eWOM influencia positivamente a utilidade da informação eWOM. 
D. Credibilidade da Informação

A credibilidade da informação refere-se à medida em que uma informação é percebida como credível, verdadeira ou factual (Cheung et al., 2009). No contexto do eWOM vários estudos já demonstraram que este determinante influencia a adoção da informação (Chang \& Wu, 2014; Cheung et al., 2009; Fan et al., 2013; Teng, Khong, Goh, \& Chong, 2014). Por conseguinte, considerando que a utilidade da informação tem um papel mediador no modelo IACM, é proposta a seguinte hipótese:

H4: Credibilidade da informação eWOM influencia positivamente a utilidade da informação eWOM.

\section{E. Credibilidade da Fonte}

De acordo com Petty e Cacioppo (1986), a credibilidade da fonte refere-se à medida em que a fonte da informação é percebida como confiável e competente pelos recipientes da informação (Cheung et al., 2008). Assim, a credibilidade da fonte pode ser considerada em duas subdimensões, nomeadamente baseada na competência, que se refere à consideração do emissor como especialista no assunto, e baseada na confiabilidade, que se baseia na perceção de caráter e integridade da fonte da informação (Sussman \& Siegal, 2003).

A perceção da credibilidade da fonte da informação é importante no processo de comunicação, visto que nem todos os emissores são especialistas ou confiáveis (Chang \& Wu, 2014). No contexto online, quando a informação é transmitida por uma fonte com credibilidade sólida, esta poderá ser percebida como mais útil e credível (Chang \& Wu, 2014; Cheung et al., 2008; Sussman \& Siegal, 2003), e sendo que no contexto do presente estudo é considerada a plataforma do Facebook, os utilizadores têm acesso a um grande conjunto de informação pessoal exposta pela sua lista de contactos, pelo que a avaliação da credibilidade da fonte será mais acessível. Assim:

H5: A credibilidade da fonte de eWOM influencia positivamente a utilidade da informação eWOM.

\section{F. Necessidades de informação}

De acordo com Erkan e Evans (2016), as necessidades de informação já foram estudadas previamente como um motivador para o envolvimento dos indivíduos em eWOM, tendo já sido usado o conceito de "procura de conselhos" (Hennig-Thurau, Gwinner, Walsh, \& Gremler, 2004; Wolny \& Mueller, 2013) e "procura de opiniões" (Chu \& Kim, 2011). O modelo IACM utiliza esta variável como um determinante do eWOM na 
influência da intenção de compra, ao considerar que indivíduos que necessitem e procurem informação estarão mais propensos a encontrar a informação mais útil e adotá-la (Erkan \& Evans, 2016). Desta maneira:

H6: Necessidades de informação eWOM influenciam positivamente a utilidade da informação eWOM.

\section{G. Atitude em relação à informação}

A atitude do recetor em relação à informação é acrescentada no modelo IACM tendo em conta o modelo TRA. De acordo com Erkan e Evans (2016), a atitude em relação à informação eWOM tem um impacto sobre a perceção da utilidade da informação e a intenção de compra do consumidor. Portanto, em conformidade com o modelo IACM:

H7: Atitude em relação à informação eWOM influencia positivamente a utilidade da informação eWOM.

H8: Atitude em relação à informação eWOM influencia positivamente a intenção de compra eWOM.

\section{H. Efeito mediador da credibilidade da informação}

No contexto da informação exposta na internet, Wathen e Burkell (2002) sugerem que os fatores da fonte (credibilidade da fonte) e da mensagem (qualidade da informação) terão importantes efeitos na avaliação da credibilidade da informação.

De facto, de acordo com Cheung et al. (2009), no contexto do eWOM, acredita-se que os recetores fazem julgamentos quanto à credibilidade da informação com base na qualidade da informação. Adicionalmente, Tsao e Hsieh (2015) referem que informação de maior qualidade poderá ampliar a capacidade de avaliação da credibilidade da informação por parte do leitor, aumentando ainda a sua confiança na informação exposta, podendo assim estimular mais eficazmente a intenção de compra ao serem providenciadas recomendações consideradas úteis. No contexto do eWOM, esta relação entre a qualidade e credibilidade da informação já foi demonstrada em vários estudos (Cheung et al., 2009; Fan et al., 2013; Teng et al., 2014; Tsao \& Hsieh, 2015).

Quanto à credibilidade da fonte, este determinante já foi também examinado como influente na adoção da informação (Zhang \& Watts, 2008), no entanto no estudo de Chang e Wu (2014), a credibilidade da fonte não demonstra influência direta na adoção da informação, sendo que estes argumentam que a credibilidade da fonte contribuirá para a sustentação da credibilidade da informação. Vários autores já demonstraram a influência exercida pela credibilidade da fonte na 
credibilidade da informação (Chang \& Wu, 2014; Cheung et al., 2009; Fan et al., 2013; Fang, 2014). Luo et al. (2013) mencionam que uma fonte altamente credível poderá aumentar diretamente a perceção de credibilidade da informação por parte dos recetores, sendo que King et al. (2014) reforçam este argumento referindo que, para além do conteúdo da mensagem, as características da fonte tendem a ser importantes na avaliação da credibilidade da informação eWOM e a sua utilidade. Deste modo, são propostas as seguintes hipóteses:

H9a: Credibilidade da informação eWOM medeia a relação entre a qualidade da informação eWOM e a utilidade da informação eWOM.

H9b: Credibilidade da informação eWOM medeia a relação entre a credibilidade da fonte de eWOM e a utilidade da informação eWOM.

\section{Figura 5}

Modelo de investigação com as respetivas hipóteses

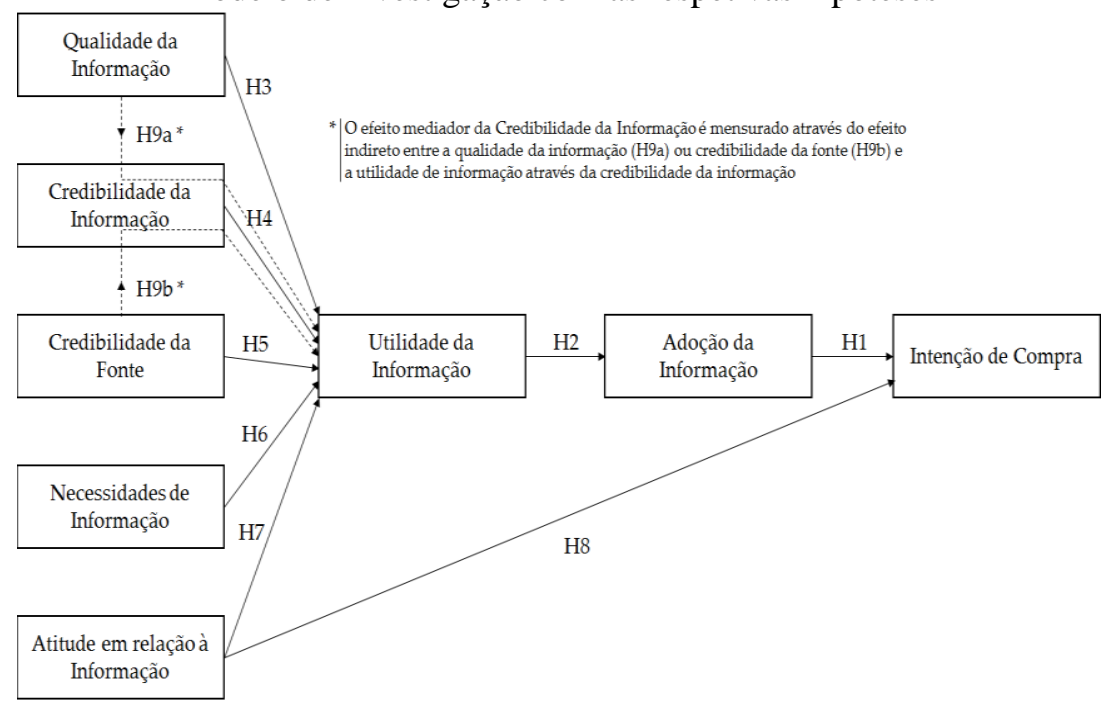

\section{METODOLOGIA DE INVESTIGAÇÃO}

Tendo em conta que o modelo de investigação adotado foi desenvolvido e testado no Reino Unido, juntamente com o facto de ser, no presente estudo, acrescentada uma variável ao mesmo e testadas hipóteses adicionais, a pesquisa aqui conduzida pode ser considerada exploratória. 


\subsection{Inquérito por questionário}

De modo a obter dados para o teste de hipóteses foi conduzido um inquérito por questionário online, elaborado através do Google Forms. Este inclui três partes. A primeira descreve sucintamente o âmbito e objetivo do questionário, incluindo uma questão filtro. A segunda contém vinte e três questões relativas às variáveis propostas. A terceira parte é relativa ao perfil demográfico dos inquiridos.

Este estudo faz uso de escalas validadas de estudos prévios, sendo todas adotadas de Erkan e Evans (2016), à exceção da variável acrescentada - credibilidade da fonte - adaptada de Sussman e Siegal (2003) (Apêndice I). Todos os itens foram avaliados com uma escala de Likert de 5 pontos, variando de discordo fortemente (1) a concordo fortemente (5). As medidas originais foram traduzidas do inglês para português, com a subsequente revisão por um elemento externo ao estudo certificado com nível de proficiência em inglês. Posteriormente, foi realizado um teste de análise semântica com sete indivíduos, cujo feedback levou a algumas alterações com vista reduzir algumas ambiguidades do questionário, aumentando a sua adequação.

\subsection{Amostra}

O questionário foi dirigido aos utilizadores do Facebook residentes em Portugal, de idades superior a 18 anos. A coleta de dados deu-se através da partilha do questionário no Facebook, tendo o processo levado duas semanas com a obtenção de 329 respostas. Após remoção de respostas que não passaram na questão filtro, de inquiridos não residentes em Portugal e padrões de resposta suspeitos, foi obtida uma amostra utilizável de 294 inquiridos para análise empírica. No Quadro 3 são apresentadas as características da amostra.

\subsection{Análise Estatística}

Este estudo recorreu à técnica de modelação de equações estruturais baseada em mínimos quadrados parciais (PLS-SEM), com recurso ao programa SmartPLS versão 3.2.6. Sendo este um método estatístico não paramétrico, oferece maior flexibilidade, não assumindo pressupostos de normalidade da distribuição dos dados, acomodando de forma mais eficaz modelos complexos e conseguindo mais facilmente incorporar constructos com um baixo número de indicadores. Adicionalmente, de acordo com Chin (2010) esta técnica é adequada em estudos incrementais, que, tal como o presente, adicionam medidas e caminhos estruturais a modelos previamente estabelecidos. 


\section{Quadro 3}

Caracterização da amostra

\begin{tabular}{lrr}
\hline Medida & Frequência & Percentagem \\
\hline Género & 175 & \\
Feminino & 119 & 59,5 \\
Masculino & & 40,5 \\
Idade & 175 & \\
$18-25$ & 48 & 59,5 \\
$26-40$ & 34 & 16,3 \\
$41-55$ & 37 & 11,6 \\
$>55$ & & 12,6 \\
Habilitações Académicas & 65 & \\
Ensino Secundário & 139 & 22,1 \\
Licenciatura & 10 & 47,3 \\
Pós-graduação & 70 & 3,4 \\
Mestrado & 6 & 23,8 \\
Doutoramento & 4 & 2,0 \\
Outros & & 1,3 \\
Distrito de Residência & 29 & \\
Lisboa & 220 & 9,9 \\
Porto & 18 & 74,8 \\
Braga & 3 & 6,1 \\
Setúbal & 12 & 1,0 \\
Aveiro & 12 & 4,1 \\
Outros & & 3,9 \\
Uso médio diário do Facebook & 82 & 27,9 \\
<1h & 107 & 36,4 \\
1-2h & 69 & 23,5 \\
2-4h & 19 & 6,5 \\
4-6h & 12 & 4,1 \\
6-8h & 2 & 0,7 \\
O8h & 3 & 0,9 \\
Outro & &
\end{tabular}

\section{ANÁLISE DOS DADOS}

A avaliação do modelo de investigação consiste em dois passos principais: a avaliação do modelo de medidas (externo), e do modelo estrutural (interno) (Hair et al., 2014).

\subsection{Avaliação do Modelo de Medidas}

Tendo em conta que o modelo apresenta medidas reflexivas, a análise do modelo de medidas passa pela avaliação da fiabilidade da consistência interna, da validade convergente, e da validade discriminante (Hair et al., 2014). 
Todos os valores do alfa de Cronbach e da fiabilidade composta são superiores a 0,70, sugerindo uma consistência interna aceitável. Em segundo lugar, os valores dos loadings standardizados são todos superiores a 0,70 , demonstrando a existência da fiabilidade individual dos indicadores e sugerindo validade convergente. Por último, a existência de validade convergente é reforçada com os valores da variância média extraída (AVE) de todas as variáveis, sempre superiores a 0,50.

\section{Quadro 4}

Avaliação da fiabilidade e da validade convergente

\begin{tabular}{|c|c|c|c|c|}
\hline Medida & $\begin{array}{c}\text { Alfa de } \\
\text { Cronbach }\end{array}$ & $\begin{array}{c}\text { Fiabilidade } \\
\text { Composta }\end{array}$ & $\begin{array}{c}\text { Outer } \\
\text { Loadings }\end{array}$ & AVE \\
\hline Qualidade da Informação & 0,754 & 0,854 & & 0,661 \\
\hline QIn1 & & & 0,808 & \\
\hline QIn2 & & & 0,810 & \\
\hline Qin3 & & & 0,821 & \\
\hline Credibilidade da & 0,898 & 0,929 & & 0,766 \\
\hline \multicolumn{5}{|l|}{ Informação } \\
\hline CIn1 & & & 0,875 & \\
\hline $\operatorname{CIn} 2$ & & & 0,900 & \\
\hline$C \operatorname{In} 3$ & & & 0,882 & \\
\hline CIn4 & & & 0,843 & \\
\hline Credibilidade da Fonte & 0,776 & 0,870 & & 0,690 \\
\hline$C F 1$ & & & 0,882 & \\
\hline$C F 2$ & & & 0,790 & \\
\hline$C F 3$ & & & 0,818 & \\
\hline Necessidades de Informação & 0,813 & 0,914 & & 0,842 \\
\hline NIn1 & & & 0,927 & \\
\hline NIn2 & & & 0,908 & \\
\hline Atitude em Relação à & 0,865 & 0,917 & & 0,788 \\
\hline \multicolumn{5}{|l|}{ Informação } \\
\hline ARIn1 & & & 0,803 & \\
\hline$A R \operatorname{In} 2$ & & & 0,945 & \\
\hline ARIn3 & & & 0,908 & \\
\hline Utilidade da Informação & 0,865 & 0,936 & & 0,880 \\
\hline UIn1 & & & 0,948 & \\
\hline UIn2 & & & 0,947 & \\
\hline Adoção da Informação & 0,916 & 0,960 & & 0,923 \\
\hline $\operatorname{AdIn} 1$ & & & 0,963 & \\
\hline $\operatorname{AdIn} 2$ & & & 0,958 & \\
\hline Intenção de Compra & 0,894 & 0,926 & & 0,758 \\
\hline ICol & & & 0,868 & \\
\hline ICo2 & & & 0,897 & \\
\hline ICo3 & & & 0,854 & \\
\hline ICo4 & & & 0,863 & \\
\hline
\end{tabular}


Quanto à confirmação da existência de validade discriminante, utilizaram-se três critérios, nomeadamente: a examinação dos cross loadings (Apêndice II); o critério de Fornell-Larcker (Apêndice III); o rácio das correlações heterotrait-monotrait (HTMT). Os resultados obtidos com os dois primeiros critérios sugerem a existência de validade discriminante dos constructos do modelo (Apêndices II e III). Quanto ao HTMT, Henseler et al. (2015) sugerem como limite máximo o valor de 0,90 para se considerar a existência de validade discriminante. Os resultados sugerem validade discriminante entre todos os construtos, exceto entre atitude em relação à informação e adoção da informação (Quadro 5).

\section{Quadro 5}

Rácio das correlações heterotrait-monotrait (HTMT)

\begin{tabular}{lccccccc}
\hline & 1 & 2 & 3 & 4 & 5 & 6 & 7 \\
\hline 1. Adoção da I. & & & & & & & \\
2. Atitude Relação à I. & $\mathbf{0 . 9 1 1}$ & & & & & & \\
3. Credibilidade Fonte & 0.554 & 0.530 & & & & & \\
4. Credibilidade da I. & 0.586 & 0.549 & 0.885 & & & & \\
5. Intenção de Compra & 0.781 & 0.778 & 0.542 & 0.561 & & & \\
6. Necessidades de I. & 0.826 & 0.875 & 0.464 & 0.555 & 0.725 & & \\
7. Qualidade da I. & 0.472 & 0.438 & 0.752 & 0.836 & 0.463 & 0.499 & \\
8. Utilidade da I. & 0.882 & 0.831 & 0.610 & 0.641 & 0.762 & 0.793 & 0.527 \\
\hline
\end{tabular}

Uma das possíveis soluções ao lidar com problemas de validade discriminante passa pela redução da média das correlações dos indicadores entre os diferentes constructos, através da eliminação de indicadores que correlacionem fortemente com indicadores de outro constructo (Henseler et al., 2015). Deste modo, procedeu-se à eliminação do indicador da escala de atitude em relação à informação (ARIn3) que apresentava o maior nível de correlação com o constructo adoção da informação. No Quadro 6, são apresentados os valores do HTMT após a alteração, confirmando a existência de validade discriminante entre todos os construtos. 


\section{Quadro 6}

Rácio das correlações heterotrait-monotrait (HTMT) após eliminação do indicador ARIn3

\begin{tabular}{lccccccc}
\hline & 1 & 2 & 3 & 4 & 5 & 6 & 7 \\
\hline 1. Adoção da I. & & & & & & & \\
2. Atitude Relação à I. & 0.863 & & & & & & \\
3. Credibilidade Fonte & 0.554 & 0.483 & & & & & \\
4. Credibilidade da I. & 0.586 & 0.479 & 0.885 & & & & \\
5. Intenção de Compra & 0.781 & 0.747 & 0.542 & 0.561 & & & \\
6. Necessidades de I. & 0.826 & 0.853 & 0.464 & 0.555 & 0.725 & & \\
7. Qualidade da I. & 0.472 & 0.390 & 0.752 & 0.836 & 0.463 & 0.499 & \\
8. Utilidade da I. & 0.882 & 0.773 & 0.610 & 0.641 & 0.762 & 0.793 & 0.527 \\
\hline
\end{tabular}

\subsection{Avaliação do Modelo Estrutural}

\section{Quadro 7}

Resultados dos coeficientes de caminho, significância e tamanhos do efeito

\begin{tabular}{|c|c|c|c|c|}
\hline Caminho & $\begin{array}{l}\text { Coeficiente de } \\
\text { caminho }(\beta)\end{array}$ & t-value & $\frac{f^{2}}{2}$ & Resultado \\
\hline $\begin{array}{l}\text { Adoção da Informação -> } \\
\text { Intenção de Compra }\end{array}$ & 0,510 & 7,179 & 0,234 & H1 Suportada*** \\
\hline $\begin{array}{l}\text { Utilidade da Informação -> } \\
\text { Adoção da Informação }\end{array}$ & 0,789 & 31,871 & 1,648 & H2 Suportada*** \\
\hline $\begin{array}{l}\text { Qualidade da Informação -> } \\
\text { Utilidade da Informação }\end{array}$ & 0,017 & 0,311 & 0,000 & H3 Não suportada \\
\hline $\begin{array}{l}\text { Credibilidade da Informação } \\
\text {-> Utilidade da Informação }\end{array}$ & 0,188 & 2,823 & 0,027 & H4 Suportada** \\
\hline $\begin{array}{l}\text { Credibilidade da Fonte -> } \\
\text { Utilidade da Informação }\end{array}$ & 0,111 & 1,804 & 0,013 & H5 Não suportada \\
\hline $\begin{array}{l}\text { Necessidades de Informação } \\
\text {-> Utilidade da Informação }\end{array}$ & 0,302 & 5,465 & 0,101 & H6 Suportada*** \\
\hline $\begin{array}{l}\text { Atitude em Relação a } \\
\text { Informação -> Utilidade da } \\
\text { Informação }\end{array}$ & 0,324 & 5,491 & 0,123 & H7 Suportada*** \\
\hline $\begin{array}{l}\text { Atitude em Relação a } \\
\text { Informação -> Intenção de } \\
\text { Compra }\end{array}$ & 0,260 & 3,466 & 0,061 & H8 Suportada** \\
\hline $\begin{array}{l}\text { Qualidade da Informação -> } \\
\text { Credibilidade da Informação }\end{array}$ & 0,419 & 9,373 & 0,332 & H9a Quadro VIII \\
\hline $\begin{array}{l}\text { Credibilidade da Fonte -> } \\
\text { Credibilidade da Informação }\end{array}$ & 0,491 & 10,537 & 0,455 & H9b Quadro VIII \\
\hline
\end{tabular}




\section{Figura 6}

Análise PLS do modelo de investigação

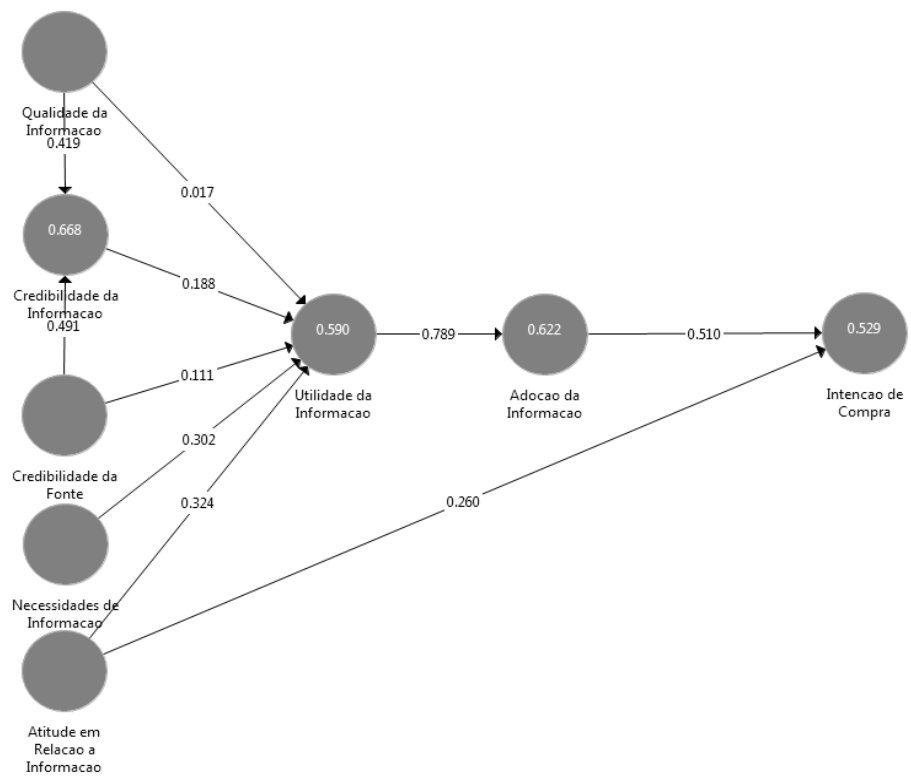

Como é possível observar no Quadro 7 e Fig. 6, de oito hipóteses das dez formuladas, seis relações são estatisticamente significantes. Mais especificamente, a adoção da informação aparenta ter um impacto positivo na intenção de compra $(\beta=0,510, t=7,179, p<0,001)$ sendo a $\mathrm{H} 1$ suportada. Adicionalmente, a utilidade da informação também demonstra uma influência positiva sobre a adoção da informação $(\beta=$ $0,789, t=31,871, p<0,001$ ), sendo a $\mathrm{H} 2$ suportada. No entanto a qualidade da informação não demonstra ter um impacto sobre a utilidade da informação $(\beta=0,017, t=0,311, p>0,05)$, pelo que a H3 não é suportada. A H4 é suportada, sendo que a credibilidade da informação mostra uma influência positiva sobre a utilidade da informação $(\beta=$ $0,188, t=2,823, p<0,01)$. A H5 não é suportada, sendo que a credibilidade da fonte não aparenta ter um impacto sobre a utilidade da informação $(\beta=0,111, t=1,804, p>0,05)$. Para além disso, a necessidade de informação e a atitude em relação à informação demonstram ter uma influência positiva sobre a utilidade da informação, com a atitude em relação à informação a ter também influência sobre a intenção de compra, pelo que respetivamente, a H6 $(\beta=0,302, t=5,465$, $p<0,001), \mathrm{H} 7(\beta=0,324, t=5,491, p<0,001)$ e H8 $(\beta=0,260, t=$ $3,466, p<0,01)$ são suportadas. 


\subsection{Efeitos Diretos, Indiretos e Totais}

De acordo com Zhao et al. (2010), há apenas uma condição necessária para estabelecer mediação, que é o efeito indireto entre a variável independente (qualidade da informação e credibilidade da fonte) e a variável dependente (utilidade da informação) através do mediador (credibilidade da informação), ser significativo. Assim, o Quadro 8 apresenta os resultados obtidos através do procedimento de reamostragem bootstrap (com cinco mil subamostras) de forma a verificar a possível significância do efeito mediador da credibilidade da informação (H9a e $\mathrm{H} 9 \mathrm{~b})$.

A Hipótese 9a prevê que a credibilidade da informação age como mediador entre a influência da qualidade da informação na perceção da utilidade da mesma, tendo sido suportada $\left(\beta^{\text {Indireto }}=0,079, \mathrm{t}=2,666, \mathrm{p}<\right.$ $0,01)$. A Hipótese $9 \mathrm{~b}$ que prevê que a credibilidade da informação age como mediador entre a credibilidade da fonte e a utilidade da informação foi suportada $\left(\beta^{\text {Indireto }}=0,091, \mathrm{t}=2,702, \mathrm{p}<0,01\right)$. Adicionalmente, visto o efeito direto da qualidade da informação e da credibilidade da fonte na utilidade da informação $(\beta=0,017, \mathrm{t}=0,311, \mathrm{p}>0,05$ e $\beta=0,111, \mathrm{t}=$ $1,804, \mathrm{p}>0,05$, respetivamente) não serem significativos, em ambos os casos estamos perante mediação apenas de tipo indireto, ou seja, mediação total (Zhao et al., 2010).

\section{Quadro 8}

Efeitos diretos e indiretos na Utilidade da Informação

\begin{tabular}{lllllll}
\hline Determinantes & $\begin{array}{l}\text { Efeitos } \\
\text { Diretos }\end{array}$ & $\begin{array}{l}\text { Efeitos } \\
\text { Indiretos }\end{array}$ & $\begin{array}{l}\text { t-value } \\
\text { (Efeitos } \\
\text { Indiretos) }\end{array}$ & $\begin{array}{l}\text { Intervalo de } \\
\text { Confiança }\end{array}$ & $\begin{array}{l}\text { Efeito de } \\
\text { Mediação }\end{array}$ & Resultado \\
\hline $\begin{array}{l}\text { Credibilidade } \\
\text { da Informação }\end{array}$ & $0,188^{* *}$ & - & - & - & - & - \\
$\begin{array}{l}\text { Qualidade da } \\
\text { Informação }\end{array}$ & $0,017^{\mathrm{NS}}$ & $0,079^{* *}$ & 2,666 & {$[0,025: 0,142]$} & $\begin{array}{l}\text { Mediação } \\
\text { Total }\end{array}$ & $\begin{array}{l}\text { H9a } \\
\text { Suportada** }\end{array}$ \\
$\begin{array}{l}\text { Credibilidade } \\
\text { da Fonte }\end{array}$ & $0,111^{\mathrm{NS}}$ & $0,092^{* *}$ & 2,702 & {$[0,031: 0,166]$} & $\begin{array}{l}\text { Mediação } \\
\text { Total }\end{array}$ & $\begin{array}{l}\text { H9b } \\
\text { Suportada** }\end{array}$ \\
\hline
\end{tabular}

Adicionalmente, de forma a obter uma visão mais aprofundada e ir ao encontro dos objetivos deste estudo, no Quadro 9 são apresentados os efeitos diretos, indiretos e totais das variáveis exógenas na intenção de compra. 


\section{Quadro 9}

Efeitos diretos, indiretos e totais na Intenção de Compra

\begin{tabular}{|c|c|c|c|c|}
\hline Determinantes & $\begin{array}{l}\text { Efeitos } \\
\text { Diretos }\end{array}$ & $\begin{array}{l}\text { Efeitos } \\
\text { Indiretos }\end{array}$ & Efeitos Totais & $\begin{array}{l}\text { t-value } \\
\text { (Efeitos } \\
\text { Totais) }\end{array}$ \\
\hline Adoção da Informação & 0,510 & - & $0,510 * * *$ & 7,179 \\
\hline Utilidade da Informação & - & 0,402 & $0,402 * * *$ & 6,515 \\
\hline Qualidade da Informação & - & 0,039 & $0,039^{\mathrm{NS}}$ & $1,744^{\mathrm{NS}}$ \\
\hline $\begin{array}{l}\text { Credibilidade da } \\
\text { Informação }\end{array}$ & - & 0,076 & $0,076^{*}$ & 2,493 \\
\hline Credibilidade da Fonte & - & 0,082 & $0,082 * *$ & 3,177 \\
\hline $\begin{array}{l}\text { Necessidades de } \\
\text { Informação }\end{array}$ & - & 0,122 & $0,122 *$ & 4,136 \\
\hline $\begin{array}{l}\text { Atitude em Relação à } \\
\text { Informação }\end{array}$ & 0,260 & 0,131 & $0,391 * * *$ & 6,090 \\
\hline
\end{tabular}

\section{DISCUSSÃO DOS RESULTADOS E CONCLUSÃO}

Os resultados obtidos no estudo empírico sugerem que, no contexto do Facebook, tanto as características do recetor, como as da fonte e as da informação exercem um impacto positivo sobre a intenção de compra. Mais especificamente, foi possível observar que o comportamento do consumidor relativamente à informação eWOM, ou seja, as características do recetor (atitude em relação à informação e necessidades de informação) são as que apresentam maior influência nesse impacto. Mais especificamente, observa-se que todas as variáveis do modelo, à exceção da qualidade da informação, são determinantes do eWOM no Facebook e, indiretamente, das intenções de compra dos consumidores.

Discriminando agora as hipóteses, Cheung e Thadani (2012) identificaram a tendência da literatura em ser tido em conta apenas uma das variáveis como a atitude, intenção de compra, compra ou adoção do eWOM, não sendo geralmente estudadas as relações entre estes impactos, tendo estes proposto que consumidores que adotarem a informação eWOM terão maior intenção de compra. No presente estudo, esta relação é empiricamente demonstrada (H1), ou seja, quando os consumidores observam informação eWOM no Facebook que consideram informativa e facilitadora da tomada de decisão relativamente a um produto ou serviço, estes expressam uma maior intenção de compra.

A H2, tendo sido suportada, demonstra ser coerente com o argumento de Sussman e Siegal (2003) de que a utilidade é um antecedente chave 
nos comportamentos de adoção da informação, servindo como mediador entre o processamento da informação e a adoção da mesma, estando também em concordância com vários outros estudos que já demonstraram essa relação (Cheung et al., 2008; Erkan \& Evans, 2016; Liu \& Zhang, 2010; Sussman \& Siegal, 2003).

No entanto, ao contrário do esperado, os resultados empíricos deste estudo não suportam a relação proposta entre a qualidade da informação e a sua utilidade (rejeição de H3). Esta ocorrência poderá originar das diferenças de natureza cultural resultantes do contexto do estudo ou do ambiente tecnológico do estudo, o Facebook. Neste contexto tecnológico, o foco é na identidade dos utilizadores comparativamente a, por exemplo, fóruns, onde o foco é num determinado tópico ou interesse, sendo também um fenómeno comum no Facebook a partilha de informação em mensagens curtas (Svensson, 2011). Juntamente com o facto de que os utilizadores do Facebook não escolhem livremente os tópicos aos quais estão expostos na newsfeed (Svensson, 2011), a informação que é partilhada pela lista de contactos relativamente a produtos e serviços pode não ter o detalhe suficiente para afetar a perceção de utilidade da informação. Portanto, os utilizadores podem observar informação eWOM exposta e considerá-la clara e compreensível, com qualidade, no entanto podem considerar que esta não é suficientemente informativa e útil relativamente ao produto ou serviço em questão.

O suporte à H4 é coerente com o estudo de Erkan e Evans (2016), e também com estudos que já demonstraram a influência da credibilidade da informação na adoção da informação (Chang \& Wu, 2014; Cheung et al., 2009; Fan et al., 2013; Teng et al., 2014), tendo em conta que, com base na perspetiva do modelo utilizado, a utilidade da informação exerce um papel mediador entre estes. Os resultados obtidos sugerem que quando os indivíduos observam informação eWOM no Facebook que consideram credível, isto afetará positivamente a sua perceção da utilidade dessa informação.

Relativamente à credibilidade da fonte, não suportam este impacto direto na utilidade da informação (rejeição de H5). Tendo em conta o contexto, os consumidores não terão muitas incertezas quanto à identidade do emissor da informação, sendo que não será pela dificuldade de avaliação da fonte que a sua credibilidade não tem impacto na utilidade da informação. No entanto, apesar dos utilizadores do Facebook não escolherem livremente os tópicos aos quais estão expostos na newsfeed (Svensson, 2011), estes têm a opção de eliminar pessoas da sua newsfeed, ou seja, neste contexto há a possibilidade dos indivíduos 
"censurarem" pessoas que não considerem relevantes e credíveis da sua lista de contactos, pelo que isto poderá ajudar a que a credibilidade da fonte não seja tão importante na avaliação da utilidade da informação.

Com o suporte à H6, observa-se que os indivíduos que necessitem e procurem informação eWOM no Facebook são mais propensos a considerá-la útil, possivelmente por terem maior predisposição em processá-la.

Erkan e Evans (2016) sugerem que consumidores que têm atitudes positivas em relação às informações eWOM terão uma maior tendência em considerá-las úteis, no entanto os resultados empíricos do seu estudo não suportaram essa hipótese. Contudo, no presente estudo, esta relação foi corroborada (H7), o que poderá dever-se, novamente, a diferenças de natureza cultural e à plataforma em questão, o Facebook. Adicionalmente, tendo a H8 sido suportada, é verificada congruência com o estudo de Erkan e Evans (2016) de que a atitude em relação à informação tem um impacto direto sobre a intenção de compra dos recetores da informação eWOM no Facebook. Isto sugere que quando os consumidores têm atitudes positivas em relação à informação eWOM exposta no Facebook, a influência da informação observada deverá ser maior, aumentando a perceção da utilidade da informação e a intenção de compra.

Relativamente à credibilidade da informação, é sugerido neste estudo que esta medeia totalmente o impacto da qualidade da informação e da credibilidade da fonte, revelando o papel central de mediação da credibilidade da informação entre ambos estes determinantes e a utilidade da informação. De acordo com Savolainen (2011), as questões da qualidade e da credibilidade da informação são multifacetadas, pelo que Metzger (2007) chega a constatar que a credibilidade da informação é uma avaliação do recetor que envolve tanto julgamentos objetivos da qualidade da informação como perceções subjetivas da credibilidade da fonte. De facto, Tsao e Hsieh (2015), com base nos resultados do seu estudo, argumentam que eWOM com qualidade de informação superior pode aumentar a capacidade do leitor para fazer uma avaliação inteligente da credibilidade da informação lida, sendo que Chang e Wu (2014) também referem que a credibilidade da fonte desempenha um papel de apoiar a informação como mais credível. Alguns estudos realizados noutros contextos de eWOM demonstram que a qualidade da informação e credibilidade da fonte influenciam a adoção da informação eWOM através da perceção da credibilidade da informação (Cheung et al., 2009; Fan et al., 2013; Fang, 2014; Teng et al., 2014). Isto é coerente com os 
resultados obtidos, tendo em conta que no presente estudo é considerado um papel mediador da utilidade da informação entre o processamento da informação e a adoção da mesma. Estes resultados sugerem que os utilizadores do Facebook poderão ter uma sensação de risco reduzida não só quando a informação eWOM aparenta ter qualidade, como quando a pessoa que partilhou a informação aparenta ser credível, o que significará uma maior preponderância a considerar essa informação como credível, e por sua vez útil. Ou seja, os recetores de eWOM no Facebook não se tornam mais propensos a considerar a informação útil apenas por esta aparentar ter qualidade e/ou ser partilhada por alguém que consideram confiável. Apenas quando acreditam que a informação exposta é de facto credível e convincente, é que tenderão a considerá-la mais útil.

\subsection{Contributos Teóricos}

Este estudo utilizou como base o modelo IACM de Erkan e Evans (2016), sendo acrescentada a variável da credibilidade da fonte, o que permitiu introduzir na análise o papel do emissor de eWOM na influência da intenção de compra dos consumidores, tendo-se revelado uma determinante estatisticamente relevante. Para além disto, o modelo conceptual usado contribui ainda teoricamente quanto à influência da informação eWOM ao investigar o efeito mediador da credibilidade da informação, tendo sido verificado que esta serve como mediador total do impacto da credibilidade da fonte e da qualidade da informação na utilidade da informação.

Adicionalmente, o presente estudo contempla no seu âmbito somente uma plataforma em particular (Facebook), algo sugerido por Erkan e Evans (2016) para investigações futuras, sendo que estes autores aplicaram o modelo IACM no contexto de websites de social media em geral.

Acrescenta-se o facto de os resultados sugerirem que o modelo IACM providencia uma estrutura teórica adequada para compreender como diferentes características influenciam a utilidade da informação, a adoção da informação, e por fim, a intenção de compra. A sua capacidade explanatória é alta, tendo gerado resultados de coeficiente de determinação $\left(\mathrm{R}^{2}\right)$ para as diferentes variáveis dependentes entre $53 \mathrm{e}$ $67 \%$ (Apêndice IV), que comparam muito bem com o limite inferior de referência para um alto poder preditivo para fenómenos relativos a comportamentos dos consumidores, definido por Hair et al. (2014) nos $20 \%$. 


\subsection{Limitações e sugestões para investigação futura}

Deste estudo resultam algumas limitações que deverão ser tidas em conta para investigações futuras.

Em primeiro lugar, a maioria dos inquiridos encontra-se na faixa etária dos 18 aos 25 anos e é residente do distrito do Porto. Seria pertinente a obtenção de uma proporção de inquiridos com características demográficas mais representativas dos utilizadores do Facebook no país.

Em segundo lugar, os resultados obtidos referem-se à intenção de compra de produtos e serviços em geral. Estudos futuros deverão procurar entender a influência dos determinantes do eWOM no Facebook na intenção de compra entre diferentes categorias de produtos e serviços, identificando potenciais diferenças.

Em terceiro lugar, é necessário cautela ao extrapolar as descobertas para outras plataformas para além do Facebook, pelo que uma comparação entre diferentes plataformas poderá trazer conclusões relevantes.

Por último, o modelo conceptual utilizado explica cerca de 52,9\% da variância da intenção de compra, o que apesar de evidenciar um poder explanatório forte, sugere que poderão estar em falta importantes determinantes. Assim, no contexto do Facebook, para além da característica reconhecida anteriormente (força dos laços), futuros estudos poderão adicionar variáveis ao modelo, como por exemplo o conhecimento prévio do recetor (Cheung et al., 2009), a classificação da recomendação (número de comentários, gostos, etc...) atratividade da fonte (Fang, 2014), entre outros. Adicionalmente, apesar de se ter verificado a fiabilidade e validade das escalas de medidas utilizadas, uma extensão do número de itens por constructo poderá ser útil. Por exemplo, de acordo com Savolainen (2011), não há consenso entre investigadores sobre o âmbito e significado dos conceitos da qualidade e credibilidade da informação, sendo que a qualidade da informação pode conter diferentes dimensões. Deste modo, há certas dimensões possíveis de ser acrescentadas que poderão reforçar o poder explanatório do modelo, como por exemplo a abrangência da informação e a compreensibilidade (Cheung et al., 2008).

\section{REFERÊNCIAS BIBLIOGRÁFICAS}

Abbasi, M. A., \& Liu, H. (2013). Measuring user credibility in social media. In A. M. Greenberg, W. G. Kennedy, \& N. D. Bos (Eds.), Social Computing, Behavioral-Cultural Modeling and Prediction. Lecture Notes 
in Computer Science (Vol. LNCS 7812, pp. 441-448). Heidelberg: Springer-Verlag. http://doi.org/10.1007/978-3-642-37210-0_48

Alhidari, A., \& Paswan, A. (2015). Personal level antecedents of eWOM and purchase intention, on social networking sites. Journal of Customer Behaviour, 14(2), 107-125. http://doi.org/10.1362/147539215X14373846805707

Awad, N. F., \& Ragowsky, A. (2008). Establishing Trust in Electronic Commerce Through Online Word of Mouth: An Examination Across Genders. Journal of Management Information Systems, 24(4), 101-121. http://doi.org/10.2753/MIS0742-1222240404

Balaji, M. S., Wei, K. K., \& Chong, A. Y. L. (2016). Determinants of negative word-of-mouth communication using social networking sites. Information \& Management, 53(4), 528-540. http://doi.org/10.1016/j.im.2015.12.002

Brown, J., Broderick, A. J., \& Lee, N. (2007). Word of mouth communication within online communities: Conceptualizing the online social network. Journal of Interactive Marketing, 21(3), 2-20. http://doi.org/10.1002/dir.20082

Caers, R., De Feyter, T., De Couck, M., Stough, T., Vigna, C., \& Du Bois, C. (2013). Facebook: A literature review. New Media \& Society, 15(6), 982 1002. http://doi.org/10.1177/1461444813488061

Chang, H. H., \& Wu, L. H. (2014). An examination of negative e-WOM adoption: Brand commitment as a moderator. Decision Support Systems, 59(1), 206-218. http://doi.org/10.1016/j.dss.2013.11.008

Chen, Y. L., Tang, K., Wu, C. C., \& Jheng, R. Y. (2014). Predicting the influence of users' posted information for eWOM advertising in social networks. Electronic Commerce Research and Applications, 13(6), 431439. http://doi.org/10.1016/j.elerap.2014.10.001

Cheung, C., Lee, M. K. O., \& Rabjohn, N. (2008). The impact of electronic word-of-mouth. Internet Research, 18(3), 229-247. http://doi.org/http://dx.doi.org/10.1108/10662240810883290

Cheung, M. Y., Luo, C., Sia, C. L., \& Chen, H. (2009). Credibility of Electronic Word-of-Mouth: Informational and Normative Determinants of On-line Consumer Recommendations. International Journal of Electronic Commerce, 13(4), 9-38. http://doi.org/10.2753/JEC1086-4415130402

Cheung, \& Thadani, D. (2012). The impact of electronic word-of-mouth communication: A literature analysis and integrative model. Decision Support Systems, $\quad$ 54(1), 461-470. http://doi.org/10.1016/j.dss.2012.06.008

Chin, W. W. (2010). How to Write Up and Report PLS Analyses. In V. E. Vinzi, W. W. Chin, J. Henseler, \& H. Wang (Eds.), Handbook of Partial Least Squares: Concepts, Methods and Applications (Springer H, pp. 171-193). Springer. http://doi.org/10.1007/978-3-540-32827-8

Chu, S.-C., \& Choi, S. (2011). Electronic Word-of-Mouth in Social 
Networking Sites: A Cross-Cultural Study of the United States and China. Journal of Global Marketing, 24(3), 263-281. http://doi.org/10.1080/08911762.2011.592461

Chu, S.-C., \& Kim, Y. (2011). Determinants of consumer engagement in electronic Word-Of-Mouth (eWOM) in social networking sites. International Journal of Advertising, 30(1), 47-75. http://doi.org/10.2501/IJA-30-1-047-075

Davis, F. D., Bagozzi, R., \& Warshaw, P. (1989). User Acceptance Of Computer Technology: A Comparison Of Two Theoretical Models. Management Science, 35(8), 982-1003., 35(8), 982-1003. http://doi.org/10.2307/2632151

Davis, F. (1989). Perceived usefulness, perceived ease of use, and user acceptance of information technology. MIS Quarterly, 13(3), 319-340. http://doi.org/10.1016/S0305-0483(98)00028-0

Doh, S., \& Hwang, J. (2009). How consumers evaluate eWOM (electronic word-of-mouth) messages. CyberPsychology \& Behavior, 12(2), 193197. http://doi.org/10.1089/cpb.2008.0109

Elwalda, A., Lü, K., \& Ali, M. (2016). Perceived derived attributes of online customer reviews. Computers in Human Behavior, 56, 306-319. http://doi.org/10.1016/j.chb.2015.11.051

Erkan, I., \& Evans, C. (2016). The influence of eWOM in social media on consumers' purchase intentions: An extended approach to information adoption. Computers in Human Behavior, 61(July), 47-55. http://doi.org/10.1016/j.chb.2016.03.003

Facebook. (2016a). Building a better news feed for you. Retrieved from https://newsroom.fb.com/news/2016/06/building-a-better-news-feed-foryou/

Facebook. (2016b). News feed FYI: Helping make sure you don't miss stories from friends. Retrieved from https://newsroom.fb.com/news/2016/06/news-feed-fyi-helping-make-sureyou-dont-miss-stories-from-friends/

Facebook. (2017). Facebook Stats. Retrieved from https://newsroom.fb.com/company-info/

Fan, Y.-W., \& Miao, Y.-F. (2012). Effect of Word-of-Mouth on Consumer Purchase Intention: The Perspective of Gender Differences. International Journal of Electronic Business Management, 10(3), 175-181. Retrieved from http://search.ebscohost.com/login.aspx?direct=true $\& d b=b t h \& A N=835248$ 33\& site $=$ ehost-live

Fan, Y.-W., Miao, Y.-F., Fang, Y.-H., \& Lin, R.-Y. (2013). Establishing the Adoption of Electronic Word-of-Mouth through Consumers' Perceived Credibility. International Business Research, 6(3), 58-65. http://doi.org/10.5539/ibr.v6n3p58

Fang, Y.-H. (2014). Beyond the Credibility of Electronic Word of Mouth: 
Exploring eWOM Adoption on Social Networking Sites from Affective and Curiosity Perspectives. International Journal of Electronic Commerce, 18(3), 67-102. http://doi.org/10.2753/JEC1086-4415180303

Fishbein, M., \& Ajzen, I. (1975). Belief, Attitude, Intention and Behavior: An Introduction to Theory and Research. Reading, MA: Addison Wesley. Retrieved from http://people.umass.edu/aizen/f\%26a1975.html

Goldsmith, R. E. (2006). Electronic Word-of-Mouth. In M. Khosrow-Pour (Ed.), Encyclopedia of E-Commerce, E-Government and Mobile Commerce (pp. 408-412). PA: IGI Publishing Hershey.

Hair, J. F. J., Hult, G. T. M., Ringle, C., \& Sarstedt, M. (2014). A Primer on Partial Least Squares Structural Equation Modeling (PLS-SEM). Sage Publications. http://doi.org/10.1016/j.lrp.2013.01.002

Hennig-Thurau, T., Gwinner, K. P., Walsh, G., \& Gremler, D. D. (2004). Electronic word-of-mouth via consumer-opinion platforms: What motivates consumers to articulate themselves on the Internet? Journal of Interactive Marketing, 18(1), 38-52. http://doi.org/10.1002/dir.10073

Hennig-Thurau, T., Malthouse, E. C., Friege, C., Gensler, S., Lobschat, L., Rangaswamy, A., \& Skiera, B. (2010). The Impact of New Media on Customer Relationships. Journal of Service Research, 13(3), 311-330. http://doi.org/10.1177/1094670510375460

Henseler, J., Ringle, C. M., \& Sarstedt, M. (2015). A new criterion for assessing discriminant validity in variance-based structural equation modeling. Journal of the Academy of Marketing Science, 43(1), 115-135. http://doi.org/10.1007/s11747-014-0403-8

Kim, A. J., \& Johnson, K. K. P. (2016). Power of consumers using social media: Examining the influences of brand-related user-generated content on Facebook. Computers in Human Behavior, 58, 98-108. http://doi.org/10.1016/j.chb.2015.12.047

King, R. A., Racherla, P., \& Bush, V. D. (2014). What we know and don't know about online word-of-mouth: A review and synthesis of the literature. Journal of Interactive Marketing, 28(3), 167-183. http://doi.org/10.1016/j.intmar.2014.02.001

Knoll, J. (2016). Advertising in social media: A review of empirical evidence. International Journal of Advertising, 35(2), 266-300. http://doi.org/10.1080/02650487.2015.1021898

Kozinets, R. V, de Valck, K., Wojnicki, A. C., \& Wilner, S. J. S. (2010). Networked narratives: Understanding word-of-mouth marketing in online communities. Journal of Marketing, 74(2), 71-89. http://doi.org/10.1509/jmkg.74.2.71

Ladhari, R., \& Michaud, M. (2015). eWOM effects on hotel booking intentions, attitudes, trust, and website perceptions. International Journal of Hospitality Management, 46, 36-45. http://doi.org/10.1016/j.ijhm.2015.01.010

Lee, E. J., \& Shin, S. Y. (2014). When do consumers buy online product 
reviews? Effects of review quality, product type, and reviewer's photo. Computers in Human Behavior, 31(1), 356-366. http://doi.org/10.1016/j.chb.2013.10.050

Liu, R. R., \& Zhang, W. (2010). Informational influence of online customer feedback: An empirical study. Journal of Database Marketing \& Customer Strategy Management, 17(2), 120-131. http://doi.org/10.1057/dbm.2010.11

Luo, C., Luo, X., Schatzberg, L., \& Sia, C. L. (2013). Impact of informational factors on online recommendation credibility: The moderating role of source credibility. Decision Support Systems, 56, 92-102. http://doi.org/10.1016/j.dss.2013.05.005

Macdonald, J. (2012). Privacy, professionalism, and social media in medicine. In B. A. Boateng \& E. W. Black (Eds.), Social media in medicine: The impact of online social networks in contemporary medicine (pp. 19-44). Stillwater, OK: New Forums Press.

Metzger, M. (2007). Making Sense of Credibility on the Web: Models for Evaluating Online Information and Recommendations for Future Research. Journal of the American Society for Information Science and Technology, 58(13), 2078-2091. http://doi.org/10.1002/asi

Obar, J., \& Wildman, S. (2015). Social media definition and the governance challenge: An introduction to the special issue. Telecommunications Policy, 39(9), 745-750. http://doi.org/10.1007/s10551-015-2769-z.For

Park, D. H., \& Kim, S. (2008). The effects of consumer knowledge on message processing of electronic word-of-mouth via online consumer reviews. Electronic Commerce Research and Applications, 7(4), 399410. http://doi.org/10.1016/j.elerap.2007.12.001

Park, D. H., Lee, J., \& Han, I. (2007). The Effect of On-Line Consumer Reviews on Consumer Purchasing Intention: The Moderating Role of Involvement. International Journal of Electronic Commerce, 11(4), 125148. http://doi.org/10.2753/JEC1086-4415110405

Petty, R., \& Cacioppo, J. (1986). Communication and Persuasion: Central and Peripheral Routes to Attitude Change. Springer-Verlag, New York.

Prendergast, G., Ko, D., \& Yuen, S. Y. V. (2010). Online word of mouth and consumer purchase intentions. International Journal of Advertising, 29(5), 687-708. http://doi.org/10.2501/S0265048710201427

PricewaterhouseCoopers. (2017). Total Retail Survey 2017: 10 Retailer Investments for an Uncertain Future. Retrieved from https://www.pwc.com/gx/en/industries/assets/total-retail-2017.pdf

Riegner, C. (2007). Word of Mouth on the Web: The Impact of Web 2 . 0 on Consumer Purchase Decisions. Journal of Advertising Research, December, 436-447. http://doi.org/10,2501/S0021849907070456

Rodgers, S., \& Wang, Y. (2011). Electronic Word of Mouth and Consumer Generated Content: From Concept to Application. In M. S. Eastin \& N. M. Burns (Eds.), Handbook of Research on Digital Media and 
Advertising: User Generated Content Consumption (pp. 212-231). Information Science Reference. http://doi.org/10.4018/978-1-60566-7928

Savolainen, R. (2011). Judging the Quality and Credibility of Information in Internet Discussion Forums. Journal of the Association for Information Science and Technology, 62(7), 1243-1256. http://doi.org/10.1002/asi

Sheppard, B. H., Hartwick, J., \& Warshaw, P. R. (1988). The Theory of Reasoned Action: A Meta-Analysis of Past Research with Recommendations for Modifications and Future Research. Journal of Consumer Research, 15(3), 325-343.

Shu, M., \& Scott, N. (2014). Influence of Social Media on Chinese Students' Choice of an Overseas Study Destination: An Information Adoption Model Perspective. Journal of Travel \& Tourism Marketing, 31(2), 286302. http://doi.org/10.1080/10548408.2014.873318

Sussman, S. W., \& Siegal, W. S. (2003). Informational Influence in Organizations: An Integrated Approach to Knowledge Adoption. Information Systems Research, 14(1), 47-65. http://doi.org/10.1287/isre.14.1.47.14767

Svensson, A. E. C. (2011). FACEBOOK - the Social Newspaper that Never Sleeps. University of Gothenberg.

Teng, S., Khong, K. W., Goh, W. W., \& Chong, A. L. (2014). Examining the antecedents of persuasive eWOM messages in social media. Online Information Review, 38(3), 746-768. http://doi.org/10.1108/OIR-042014-0089

Tsao, W. C., \& Hsieh, M. T. (2015). eWOM persuasiveness: do eWOM platforms and product type matter? Electronic Commerce Research, 15(4), 509-541. http://doi.org/10.1007/s10660-015-9198-z

Wang, X., Yu, C., \& Wei, Y. (2012). Social Media Peer Communication and Impacts on Purchase Intentions: A Consumer Socialization Framework. Journal of Interactive Marketing, 26(4), 198-208. http://doi.org/10.1016/j.intmar.2011.11.004

Wathen, C. N., \& Burkell, J. (2002). Believe it or not: Factors influencing credibility on the Web. Journal of the Association for Information Science and Technology, 53(2), 134-144. http://doi.org/10.1002/asi.10016

Wei, P. S., \& Lu, H. P. (2013). An examination of the celebrity endorsements and online customer reviews influence female consumers' shopping behavior. Computers in Human Behavior, 29(1), 193-201. http://doi.org/10.1016/j.chb.2012.08.005

Wolny, J., \& Mueller, C. (2013). Analysis of fashion consumers' motives to engage in electronic word of mouth communication through social media platforms. Journal of Marketing Management, 29(5-6), 562-583. http://doi.org/10.1080/0267257X.2013.778324

Yan, Q., Wu, S., Wang, L., Wu, P., Chen, H., \& Wei, G. (2016). E-WOM 
from e-commerce websites and social media: Which will consumers adopt? Electronic Commerce Research and Applications, 17, 62-73. http://doi.org/10.1016/j.elerap.2016.03.004

Zhang;, W., \& Watts, S. (2008). Capitalizing on Content: Information Adoption in Two Online communities. Journal of the Association for Information Systems, 9(2), 73-94. http://doi.org/Article

Zhang, K. Z. K., Zhao, S. J., Cheung, C., \& Lee, M. K. O. (2014). Examining the influence of online reviews on consumers' decision-making: A heuristic-systematic model. Decision Support Systems, 67(November), 78-89. http://doi.org/10.1016/j.dss.2014.08.005

Zhao, X., Lynch Jr., J. G., \& Chen, Q. (2010). Reconsidering Baron and Kenny: Myths and Truths about Mediation Analysis. Journal of Consumer Research, 37(2), 197-206. http://doi.org/10.1086/651257

Zhu, D. H., Chang, Y. P., \& Luo, J. J. (2016). Understanding the influence of $\mathrm{C} 2 \mathrm{C}$ communication on purchase decision in online communities from a perspective of information adoption model. Telematics and Informatics, 33(1), 8-16. http://doi.org/10.1016/j.tele.2015.06.001 


\section{APÊNDICES}

\section{APÊEDICE I - Medidas utilizadas para as variáveis}

Fator Itens

Sobre as informações que são partilhadas pela minha lista de contactos no Facebook sobre produtos ou serviços (através do feed de notícias, mural, chat, etc..), na minha opinião:

\begin{tabular}{|c|c|c|}
\hline $\begin{array}{l}\text { Qualidade da } \\
\text { Informação } \\
\text { (Erkan \& Evans, 2016) }\end{array}$ & $\begin{array}{l}\text { QIn2 } \\
\text { QIn3 }\end{array}$ & $\begin{array}{l}\text { Penso que são compreensíveis. } \\
\text { Penso que são claras. } \\
\text { No geral, penso que a qualidade é alta. }\end{array}$ \\
\hline $\begin{array}{l}\text { Credibilidade da } \\
\text { Informação } \\
\text { (Erkan \& Evans, 2016) }\end{array}$ & $\begin{array}{l}\text { CIn1 } \\
\text { CIn2 } \\
\text { CIn3 } \\
\text { CIn4 }\end{array}$ & $\begin{array}{l}\text { Penso que o conteúdo das mensagens que leio } \\
\text { é convincente. } \\
\text { Penso que }(\ldots) \text { é sólido. } \\
\text { Penso que }(\ldots) \text { é credível. } \\
\text { Penso que }(\ldots) \text { é preciso. } \\
\end{array}$ \\
\hline $\begin{array}{l}\text { Credibilidade da fonte } \\
\text { (Sussman \& Siegal, } \\
\text { 2003) }\end{array}$ & $\begin{array}{l}\mathrm{CFo} 1 \\
\mathrm{CFo} 2 \\
\mathrm{CFo} 3\end{array}$ & $\begin{array}{l}\text { Penso que os autores são bem informados } \\
\text { sobre o produto ou serviço. } \\
\text { Penso que os autores são especialistas sobre o } \\
\text { produto ou serviço. } \\
\text { Penso que os autores são confiáveis } \\
\end{array}$ \\
\hline $\begin{array}{l}\text { Necessidades de } \\
\text { Informação } \\
\text { (Erkan \& Evans, 2016) }\end{array}$ & $\begin{array}{l}\text { NIn1 } \\
\text { NIn2 }\end{array}$ & $\begin{array}{l}\text { Gosto de usá-las quando considero novos } \\
\text { produtos ou serviços. } \\
\text { Se tenho pouca experiência com um produto } \\
\text { ou serviço, frequentemente uso-as. }\end{array}$ \\
\hline $\begin{array}{l}\text { Atitude em Relação à } \\
\text { Informação } \\
\text { (Erkan \& Evans, 2016) }\end{array}$ & $\begin{array}{l}\text { ARIn1 } \\
\text { ARIn2 } \\
\text { ARIn3 }\end{array}$ & $\begin{array}{l}\text { Eu leio-as sempre quando compro um produto } \\
\text { ou serviço. } \\
\text { Ajudam-me na tomada de decisão quando } \\
\text { adquiro um produto ou serviço. } \\
\text { Fazem-me sentir confiante na aquisição de um } \\
\text { produto ou serviço. }\end{array}$ \\
\hline $\begin{array}{l}\text { Utilidade da Informação } \\
\text { (Erkan \& Evans, 2016) }\end{array}$ & $\begin{array}{l}\text { UIn1 } \\
\text { UIn2 } \\
\end{array}$ & $\begin{array}{l}\text { Penso que são geralmente úteis. } \\
\text { Penso que são geralmente informativas. }\end{array}$ \\
\hline $\begin{array}{l}\text { Adoção da Informação } \\
\text { (Erkan \& Evans , 2016) }\end{array}$ & $\begin{array}{l}\text { AdIn1 } \\
\text { AdIn2 }\end{array}$ & $\begin{array}{l}\text { Facilitam-me a decisão de compra. } \\
\text { Melhoram a minha eficiência na tomada de } \\
\text { decisão de compra. }\end{array}$ \\
\hline
\end{tabular}

Após considerar informações positivas sobre um produto ou serviço partilhadas pelos meus amigos no Facebook:

Intenção de Compra ICo1 É muito provável que eu adquira o produto ou serviço.

(Erkan \& Evans, 2016) $\quad$ ICo2 $\quad$ Eu vou adquirir o produto ou serviço da próxima vez que o necessitar.

ICo3 Eu vou definitivamente experimentar o produto ou serviço.

ICo4 Eu vou recomendar o produto ou serviço aos meus amigos. 
APÊEDICE II - Cross Loadings dos indicadores originais

\begin{tabular}{lllllllll}
\hline & AdIn & ARIn & CFo & CIn & ICo & NIn & QIn & UIn \\
\hline AdIn1 & 0,963 & 0,806 & 0,460 & 0,536 & 0,694 & 0,703 & 0,418 & 0,789 \\
AdIn2 & 0,958 & 0,771 & 0,437 & 0,486 & 0,665 & 0,670 & 0,368 & 0,725 \\
ARIn1 & 0,580 & 0,803 & 0,265 & 0,255 & 0,505 & 0,544 & 0,193 & 0,490 \\
ARIn2 & 0,784 & 0,945 & 0,435 & 0,494 & 0,660 & 0,727 & 0,388 & 0,705 \\
ARIn3 & 0,797 & 0,908 & 0,456 & 0,539 & 0,658 & 0,686 & 0,401 & 0,732 \\
CFo1 & 0,407 & 0,398 & 0,882 & 0,691 & 0,383 & 0,351 & 0,568 & 0,448 \\
CFo2 & 0,376 & 0,348 & 0,790 & 0,509 & 0,384 & 0,273 & 0,446 & 0,359 \\
CFo3 & 0,382 & 0,361 & 0,818 & 0,642 & 0,360 & 0,297 & 0,499 & 0,438 \\
CIn1 & 0,473 & 0,430 & 0,644 & 0,875 & 0,465 & 0,446 & 0,636 & 0,530 \\
CIn2 & 0,482 & 0,456 & 0,661 & 0,900 & 0,461 & 0,418 & 0,654 & 0,488 \\
CIn3 & 0,474 & 0,454 & 0,649 & 0,882 & 0,411 & 0,392 & 0,587 & 0,509 \\
CIn4 & 0,435 & 0,412 & 0,663 & 0,843 & 0,425 & 0,406 & 0,641 & 0,455 \\
ICo1 & 0,597 & 0,577 & 0,329 & 0,399 & 0,868 & 0,546 & 0,319 & 0,557 \\
ICo2 & 0,631 & 0,636 & 0,367 & 0,437 & 0,897 & 0,572 & 0,355 & 0,601 \\
ICo3 & 0,593 & 0,584 & 0,395 & 0,409 & 0,854 & 0,509 & 0,323 & 0,569 \\
ICo4 & 0,642 & 0,609 & 0,473 & 0,506 & 0,863 & 0,531 & 0,406 & 0,609 \\
NIn1 & 0,686 & 0,722 & 0,362 & 0,458 & 0,598 & 0,927 & 0,388 & 0,646 \\
NIn2 & 0,624 & 0,636 & 0,318 & 0,413 & 0,537 & 0,908 & 0,362 & 0,580 \\
QIn1 & 0,289 & 0,266 & 0,417 & 0,478 & 0,244 & 0,298 & 0,808 & 0,288 \\
QIn2 & 0,273 & 0,254 & 0,376 & 0,500 & 0,269 & 0,260 & 0,810 & 0,283 \\
QIn3 & 0,405 & 0,379 & 0,633 & 0,713 & 0,424 & 0,404 & 0,821 & 0,478 \\
UIn1 & 0,779 & 0,758 & 0,468 & 0,555 & 0,637 & 0,675 & 0,436 & 0,947 \\
UIn2 & 0,697 & 0,614 & 0,478 & 0,505 & 0,623 & 0,576 & 0,411 & 0,930 \\
\hline & & & & & & & &
\end{tabular}


APÊNDICE III - Critério de Fornell-Larcker dos indicadores originais

\begin{tabular}{|l|l|l|l|l|l|l|l|l|}
\hline & AIn & ARI & CF & CI & IC & NI & QI & UI \\
\hline Adoção da Informação (AIn) & $\mathbf{0 , 9 6 1}$ & & & & & & & \\
\hline Atitude em Relação a Informação (ARI) & 0,822 & $\mathbf{0 , 8 8 8}$ & & & & & & \\
\hline Credibilidade da Fonte (CF) & 0,467 & 0,445 & $\mathbf{0 , 8 3 1}$ & & & & & \\
\hline Credibilidade da Informação (CI) & 0,533 & 0,500 & 0,747 & $\mathbf{0 , 8 7 5}$ & & & & \\
\hline Intenção de Compra (IC) & 0,708 & 0,691 & 0,450 & 0,504 & $\mathbf{0 , 8 7 1}$ & & & \\
\hline Necessidades de Informação (NI) & 0,715 & 0,742 & 0,372 & 0,475 & 0,620 & $\mathbf{0 , 9 1 8}$ & & \\
\hline Qualidade da Informação (QI) & 0,410 & 0,382 & 0,612 & 0,719 & 0,404 & 0,409 & $\mathbf{0 , 8 1 3}$ & \\
\hline Utilidade da Informação (UI) & 0,789 & 0,736 & 0,503 & 0,566 & 0,671 & 0,670 & 0,452 & $\mathbf{0 , 9 3 8}$ \\
\hline
\end{tabular}

APÊEDICE IV - Precisão de Relevância preditiva (R2 e Q2)

\begin{tabular}{|l|l|l|}
\hline & $\mathrm{R}^{2}$ & $\mathrm{Q}^{2}$ \\
\hline Adoção da Informação & 0,622 & 0,558 \\
\hline Credibilidade da Informação & 0,668 & 0,493 \\
\hline Intenção de Compra & 0,529 & 0,385 \\
\hline Utilidade da Informação & 0,590 & 0,493 \\
\hline
\end{tabular}

